\title{
Mechanical and Thermal Analysis of the Spectroscopy X-ray Telescopes for the Constellation-X Mission
}

\author{
Kai-Wing Chan*a,b, Jeffrey Bolognese ${ }^{\mathrm{b}}$, Timo Saha ${ }^{\mathrm{b}}$, James Sturm ${ }^{\mathrm{b}}$, William Zhang ${ }^{\mathrm{b}}$ \\ ${ }^{a}$ Center for Research and Exploration in Space Science and Technology / \\ University of Maryland, Baltimore County, 1000 Hilltop Circle, Baltimore, MD, USA 21250; \\ ${ }^{\mathrm{b}}$ NASA/Goddard Space Flight Center, Greenbelt, MD USA 20771
}

\begin{abstract}
Area and mass requirements for the Constellation-X Spectroscopy X-Ray Telescopes restrict the thickness of the mirror segment to below a mm. Requirement of angular resolution of 15" over the soft X-ray band implies that allowable optic deformation is sub-micrometer for these thin segments. These requirements place stringent constraint on the mounting, alignment and affixing of these mirror segments in both the metrology and integration processes. We present analyses and optimization of the Constellation-X mirrors under relevant mechanical and thermal environments.
\end{abstract}

Keywords: Constellation-X, X-Ray Telescope, Segmented Optics, Gravity Sag, Thermal Deformation.

\section{INTRODUCTION}

Constellation-X is an X-ray observatory dedicated to high-resolution X-ray spectroscopy, providing over $6000 \mathrm{~cm}^{2}$ of effective area at $6 \mathrm{keV}$ and over $15000 \mathrm{~cm}^{2}$ at $1.25 \mathrm{keV}$. This high throughput is attained using 4 identical, co-aligned, high-throughput Spectroscopy X-ray Telescopes (SXT), each having a $10 \mathrm{~m}$ focal length and is $1.3 \mathrm{~m}$ in diameter. (For a general overview and recent SXT technology development, see ref. 1, 2.) In the current design, each SXT consists of 163 shells of segmented thin mirrors, focusing x-rays in two reflections at grazing incidence. The telescopes will be built in a modular fashion. In addition to the pre-collimation and post-collimation modules, each of the 4 Flight Mirror Assemblies, the optical component of the SXT, consists of 2600 mirror segments reflecting $\mathrm{x}$-ray at grazing incidence. To achieve over $200 \mathrm{~m}^{2}$ of physical surface area for such grazing incident reflection within a reasonable mass limit, the area density of the Constellation-X SXT mirror segments is limited to about $1 \mathrm{~kg} / \mathrm{m}^{2}$. We have chosen Schott glass D263, with a density of $2.5 \mathrm{~g} / \mathrm{cm}^{3}$ and a thickness of $0.4 \mathrm{~mm}$, as the mirror substrate to provide the needed areal density for this mission.

One of the technical challenges in the development of these thin mirror segments, in addition to their fabrication to the required figure precision, is their proper mounting for metrology and for flight ${ }^{3,4}$. In the current design, the mirrors are $20 \mathrm{~cm}$ long axially, and 36 degrees (outer mirrors) or 72 degrees (inner mirrors) azimuthally. To an approximation, a typical mirror is a thin cylindrical glass shell with a thickness of $0.4 \mathrm{~mm}$, a length or height of $20 \mathrm{~cm}$, and an arc length ranging from about 20 to $40 \mathrm{~cm}$, depending on the radius of the mirror. Such a mirror can easily be subjected to distortion under gravity in ground test, from thermal stress due to temperature variation or coating stress from deposition of metallic film for x-ray reflection. In this paper, we address some of the issues in ground metrology test, alignment and assembly, for the mounting configurations we considered and used. Discussion of coating stress will not be covered in this paper.

\subsection{Definitions, Nomenclature and Scope}

In this paper, we will illustrate the issues associated with mounting distortion typically of a mirror with a nominal diameter of $489 \mathrm{~mm}$, and an angular span of 50 degrees. The angular size reflects that of the mirrors in the current fabrication runs. The arc length of this mirror is about $213 \mathrm{~mm}$ (with slight differences, due to the conical geometry, between the fore and aft ends of the mirror). With a $200 \mathrm{~mm}$ axial length, the mirror is actually not too different from a square when viewed from the front surface. The designed axial sag for this mirror is about 1.1 micrometers.

To facilitate discussion, we use the following nomenclature. We shall refer to the three dimensions of a mirror in its natural cylindrical terms: axial, azimuthal and radial. The boundaries in the axial directions are referred to as axial ends, 
or simply, ends. The end with a larger radius, where the incident $\mathrm{x}$-ray may enter, is the fore end; while the one with a smaller radius, where x-ray exits, is the aft end. The boundaries in the azimuthal direction is referred to as azimuthal edges, or simply, edges. Generally, there is no distinction between the two edges. For the purpose of this paper, since we will focus mostly on the axial sag of the mirror figure but not on higher order distortion, there will be no specific distinction between a hyperbolic or parabolic axial figure, for mirrors in the primary or secondary stage.

We will discuss, in this paper, mirror distortion due to gravity or thermal strain by finite element modeling. Especially, we will address low order axial figure distortion. Distortion with smaller spatial scale related to other processes or characteristics, such as from the fabrication processes or mirror surface micro-roughness, is not our concern here. This, of course, does not mean that higher order figure distortion is unimportant.

\subsection{Mirror Orientation}

We have investigated gravity and other mirror distortions under both mirror "horizontal" and mirror "vertical" mounting configurations. The vertical and horizontal configurations refer to the general orientation of the optical axis of the mirror segment. These mounts are primarily metrology mounts but we study them with an eye towards an understanding of the mounting in a permanent flight setting.

To test of mirrors in the $\mathrm{x}$-ray, the mirror is presently required to be in horizontal configuration. We use a point $\mathrm{x}$-ray source at a distance to create a near parallel $x$-ray beam at the mirror aperture. As of now, our facility has a beam line of $600 \mathrm{~m}$ and it naturally demands a horizontal configuration. To achieve a distortion-free mounting of the thin mirrors, we built and studied a "mattress" mount, which is used both as a temporary mount for metrology as well as a mounting system for the eventual transfer to a rigid structure. A "mattress" mount is one in which the mirror is placed with its back on a bed of soft coils that are anchored on a near-cylindrical (conical) cradle. The transfer to a rigid structure is by bonding a mirror on the mattress in its minimally distortion state, to a thermally and structurally compatible frame we called a 'cube'.

In addition to mirror in a horizontal orientation, we also have studied mirror in a vertical configuration. A mirror in vertical orientation generally suffers smaller gravity sag, as the force no longer acts perpendicular to the mirror surface. This has distinct advantage in the alignment of mirrors and their integration into the telescope housing. The orientation is also somewhat simpler in normal incident metrology. For this orientation, we have studied a simple 3-point kinematic mount and a modified 4-point "cantor-tree" mount. In this paper, however, we will concentrate on addressing the mirror in the horizontal orientation. Discussion for the vertical orientation will be presented elsewhere.

In the following section, we will discuss issues associated with the horizontal orientation of the mirror axis and mirror facing up. Configuration with mirror facing sideway can be a configuration with different distortions. Such configuration will not be discussed in the present paper. We will address issues such as the general characteristics of gravity distortion of a mirror, its depends on the size of the mirror, the mattress/cradle support system and its performance, transfer of mirror to a rigid structure, and associated thermal and dynamical effects.

\section{GENERAL CONSIDERATION}

A typical Constellation-X glass mirror, if supported horizontally with a small number of fixed boundary points, will introduce substantial gravity sag. To illustrate this point, we note that the magnitude of gravity sag for a horizontal beam or flat plate with rectangular cross-section, supported at or near the ends, is

$$
\Delta=\frac{f}{32} \frac{\rho g}{E} \frac{L^{4}}{\tau^{2}}
$$

where $\Delta$ is the maximum sag near the middle of the plate, $\rho$ the density of the material, $g$ the acceleration due to gravity, $E$ the Young's modulus of the material, $L$ the length and $\tau$ the thickness. The factor $f$ is typically of the order of unity, and it depends on the types of support and support locations. For example, $f$ is 1 and 5 for a beam fixed and simply supported at the ends respectively, and -0.5 and 0.1875 for a beam simply supported and fixed symmetrically at a quarter of its length from the ends (negative sag indicates that the shape of the beam is convex instead of concave upward.) For the last case, the beam is M-shaped with the maximum deflection actually at the ends, while the lesser maximum is at the middle with an $f$ value of 0.0625 . With typical dimensions of the mirror, and a Young's modulus $E=73 \mathrm{GPa}$ for the present glass of choice for the Constellation-X SXT, $\Delta$ is $0.105 \mathrm{fmm}$. This magnitude of sag is generally too large for our application even for a small value of $f$ value such as $\sim 0.1$. We should also note that with a small bond line that we 
envision desirable for the bonding of Constellation-X mirrors, the boundary conditions at the bonds are closer to a simple support with little constrain in the local rotation of the mirror, thus a very small $f$ value $<<1$ will not be justified. This kind of gravity sag from a beam illustrates the order of magnitude of the sag of mirror supported at two or a small number of points in the azimuthal direction where the approximation of a cylindrical or conical mirror to a plate is reasonable in terms of elastic deformation. The consideration also can set an upper limit to the axial sag near the edges of the mirror, where reaction from anticlastic bending of the conical/cylindrical mirror is minimal.

For a cylindrical mirror, we calculate the gravity sag with finite element models. The modeling is accomplished with the software COMSOL Multiphysics. A result is shown in figure 1, for the case of 4 side-bonds, 2 bonds at each edge of the mirror (only half of the mirror is modeled, from symmetry.) The bonds are fixed at the boundary condition. As discussed above, this may be too strict a condition than can be implemented. Two cases are illustrated, one for bond points at the corner of the mirror, one for the two bond points along an edge at a quarter of mirror length from the ends (i.e., $50 \mathrm{~mm}$ from the corners). Major deflection occurs, as expected, at the edge. For the case of the corner bond points, the axial profile has a maximum sag in the middle. For the case of $1 / 4$-length bond locations, the axial profile is " $\mathrm{M}$ "'-shaped, with a maximum deformation at the ends that is less than 0.2 of that of the first case. These trends are consistent with the sag from a beam as discussed above (where the theoretical ratio is 0.1875 ). The axial sag is much more constrained in the azimuthal center of the mirror. At any rate, gravity sag of these magnitudes are too large for the purpose of the Constellation-X mirrors, where $1 \mu \mathrm{m}$ of sag over half of the mirror length corresponds to a general slope of 2 seconds of arc, and thus a deviation of 4 seconds of arc for reflected rays.
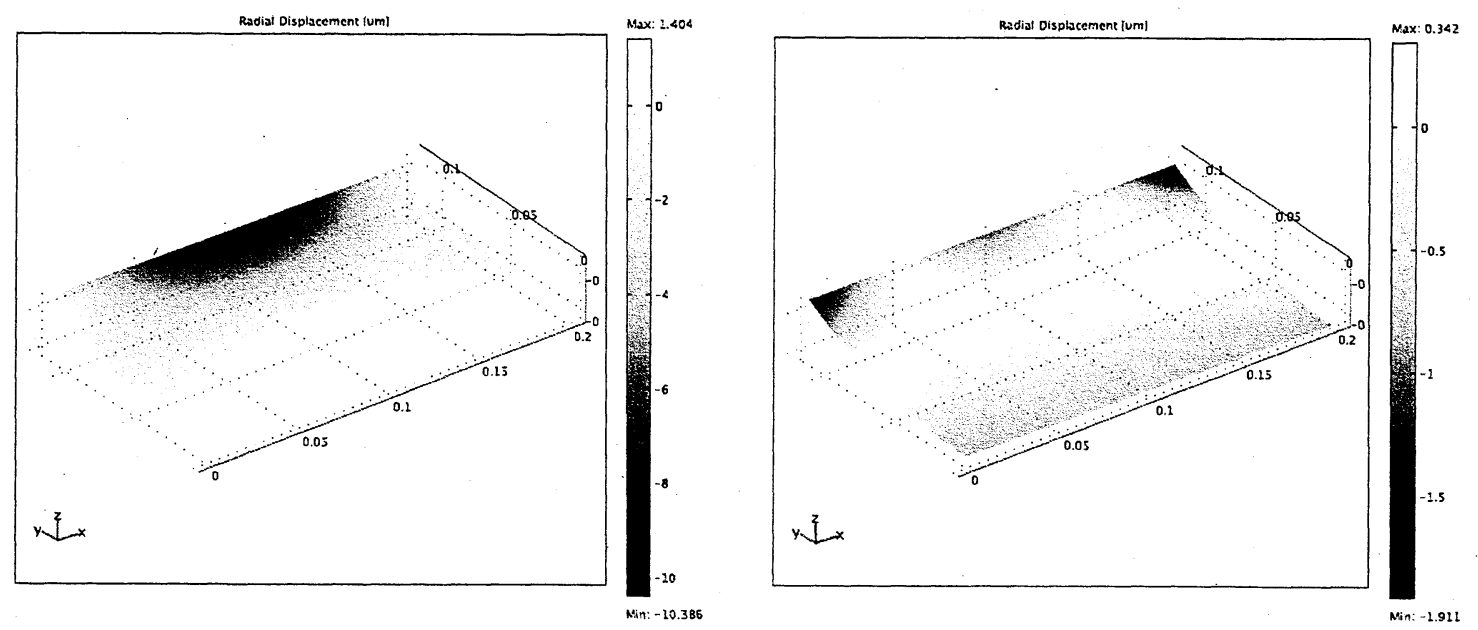

Fig. 1. Surface profile showing distortion of mirror with 4 fixed mount points at mirror edges. By symmetry, only half of the mirror is shown. Mirror axis is horizontal (i.e., gravity is in negative $\mathrm{z}$ direction.) Deformation shown is radial displacement $d R$. (Left) Bonds are at the corners of the mirror. The largest axial sag is at the edge. Anticlastic bending causes the azimuthal center of the mirror to bend upward. Axial distortion, which is important in x-ray imaging, is large $\sim 10 \mu \mathrm{m}$. (Right) Four fixed points at the side, at $5 \mathrm{~mm}$ from each end. Axial distortion is about 5 times smaller at the edge, and the profile has a characteristic $M$ shape deformation.

We optimized the bond location for the 4-point side bond by modeling a series of boundary conditions, where the bond separation is varied. The result is shown in figure 2 in which the axial profiles are plotted. For wider bond separation (> $14 \mathrm{~cm}$ ), axial profiles at the edge are simple sags with maximum deformation at the middle. The axial profile at the center of the mirror is also concave, but the displacement is generally upward. The deviation decreases with decreasing separation. For smaller bond separation $(<10 \mathrm{~cm})$, axial profiles at the edge are M-shaped with maximum deviation at the ends. Axial profiles in the middle are convex and are of smaller magnitudes. The general deformation increases as the separation is further reduced. The optimal separation is found to be $\sim 11 \mathrm{~cm}$, which is consistent with expectation from a simple beam where $\sim 55 \%$ of full length is optimal. The minimum sag under such condition is $1.5 \mu \mathrm{m}$ at the axial ends as well as at the center (the profile is, again, "M"- shaped.) We note again that the general slope of $1.5 \mu \mathrm{m}$ over 5 $\mathrm{mm}$ is still too large at 6 arc-sec. We conclude that 4-bond points at the edges will not satisfy our requirements and that more bonding points are needed, or that they are at other part of the boundary. 


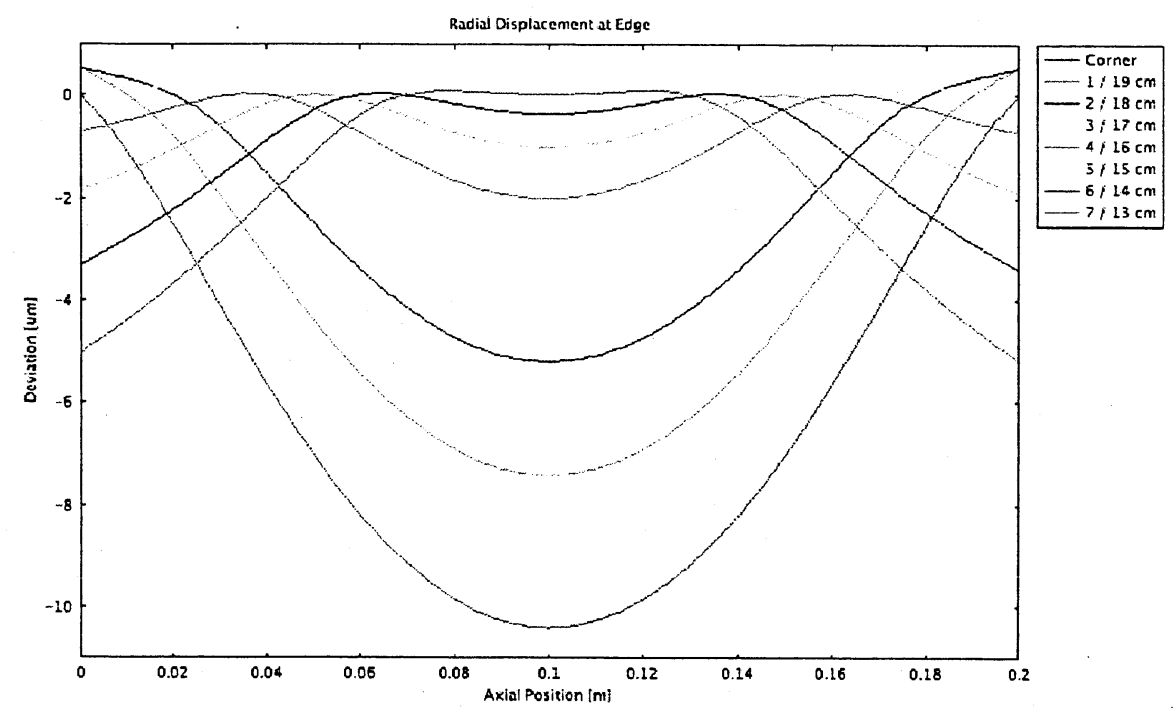

Fig. 2. Axial profile of the mirror at the edge, for 4 bond points, 2 at each end, for a series of point separations. Bond points are set axially symmetrically along the edge, for supports at the corners, and $1,2, \ldots, 7 \mathrm{~cm}$ from the ends. The curve in the gray scale figure can be distinguished from their mount points, where the displacement is zero. The mirrors with a wider separated mount points have larger sag, showing the change from simple sag with maximum deflection at the center to a $\mathrm{M}$ shape deformation with maximum deflection at the axial ends. The transition is with a separation about 0.55 of the full length. Which is also the minimum deflection. Note that $1.5 \mu \mathrm{m}$ corresponds to a general slope of $6 "$ "

An alternative configuration is to mount at the axial ends. In figure 3, we show the axial profiles at different azimuths from a finite-element model for a simple conical mirror, simply supported with 2 points at the each end for a 50 -degree mirror with a radius of $244.5 \mathrm{~mm}$ at the large end. The semi-cone angle for this mirror is $1.2^{\circ}$. The bonds are separated symmetrically in the azimuthal direction by 27.5 degree, for minimal azimuthal deviation as discussed before. The axial profiles are generally convex in the center of the mirror and in the outer part of the mirror; and concave near the azimuths where the mirror is mounted. Like the mirror with side mount, the typical sag is large for the end mounts, even though the distortion will be smaller if the mirror is further constrained also in the rotational angle at the bond points.

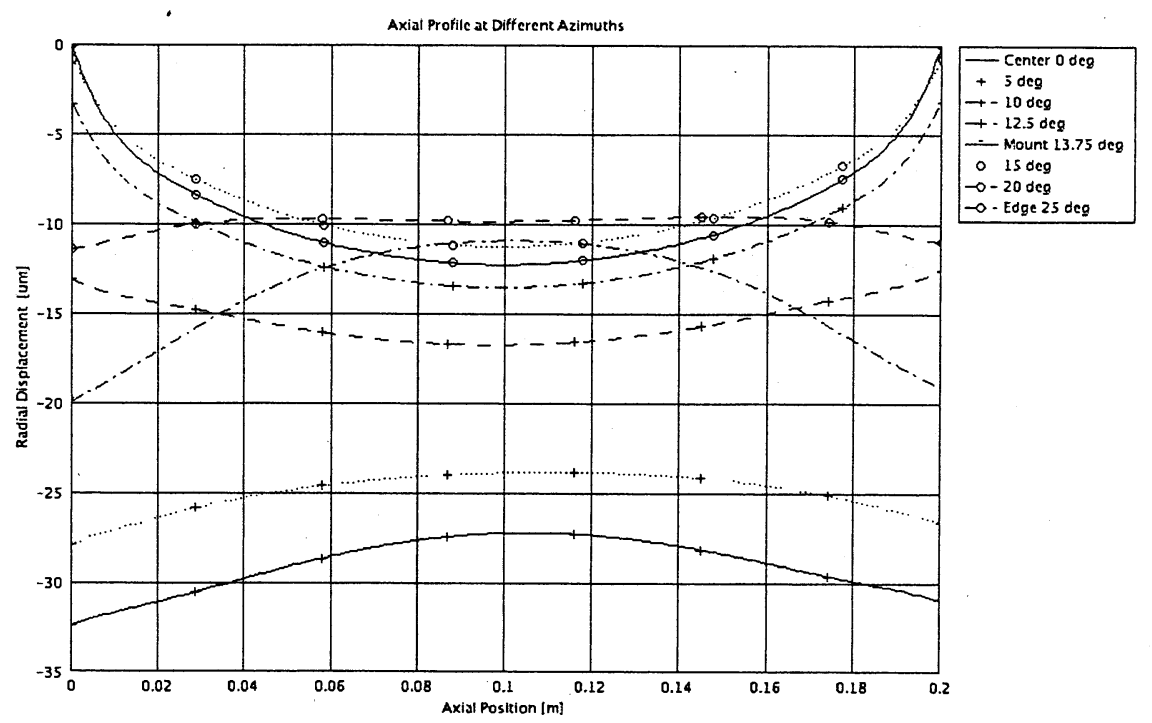

Fig. 3. Axial profiles at different azimuths for a model where the mirror is supported at 2 bond points at each end. The bonds are at $+/-13.75$ degree (a separation of 27.5 degree for the 50-degree mirror). Distortion is generally concave near the azimuths where the mirror is mounted and convex at the edges and in the center of the mirror. Typical sags are quite large at several $\mu \mathrm{m}$ up to $10 \mu \mathrm{m}$. 
To further reduce the possible gravity distortion from mounting the mirror in a horizontal orientation, a larger number of mount points can be considered. The spacing of bond points can also be optimized in both azimuthal as well as axial directions. In principle, 4 points can simultaneously be supported in its own shape if the cylinder/cone axis is properly adjusted (in what is commonly known as the yaw direction.) If more bond points are used, condition of overconstraining the mirror becomes an issue. In the case of Constellation-X, mirror bonded at more than 4 points to a rigid structure cannot tolerate incompatible bond locations even to a level of a micrometer. Multiple numbers of bonds more than 4 will not over-constrain the mirror only if the bond is not rigid, for instance, the mirror can be simply slotted or the bonding adhesive is relatively compliant. But precise alignment and unpredictable reaction force to the mirror at the bond locations will become a problem. In Section 4, we will describe a mounting mechanism where hundreds of contact points are made to mount a mirror without significantly distorting it axially.

\section{DEPENDENCE ON ANGULAR SIZE OF MIRROR}

So far, we have been discussing the gravity sag of a mirror with particular dimensions. For the Constellation-X Spectroscopy Telescopes, the radius of the mirror shell ranges from 15 to $65 \mathrm{~cm}$. Depending on the angular size of the mirror, the largest segment may be as long as $40 \mathrm{~cm}$ in the baseline design. We would need to understand how gravity distortion depends on the size and radius of the mirror. We need to answer two basic questions related to the variable of angular size: 1. For a fixed radius, what is the optimal angular span in terms of gravity deformation, according to some reasonable mounting configuration? 2. How does this optimal size scale with mirrors of different radii? For the first question, we model mirror segment of $200 \mathrm{~mm}$ in length and a radius of $R=244.5 \mathrm{~mm}$, in a 3-point mounting configuration. In this configuration, the mirror is simply support at the axial ends. One point of support is at the middle of an end of the mirror, two other points of support, separated azimuthally by 0.55 of the angular span, are at the other end. The result is shown in figure 4, in which the axial figures for the mirror at the edge and at the center are shown as a function of mirror azimuthal size, from 10 to 72 degrees. For mirror with large angular span, the axial profile at the edge is generally convex, similar to the model discussed above. Closer to the center where the mirror is mounted, the axial profiles are concave. For mirror with small angular span, the mirror is geometrically like an elongated flat thin strip and statically behaves as one, too: the mirror sags like a plate, quite uniformly across all azimuths. The cross over point turns out to be about 30 degrees, which is also the point when the sag is minimum. A $30^{\circ}$ segment corresponds to an azimuthal dimension about $0.5 R$, and an aspect ratio of about the same for width to length.

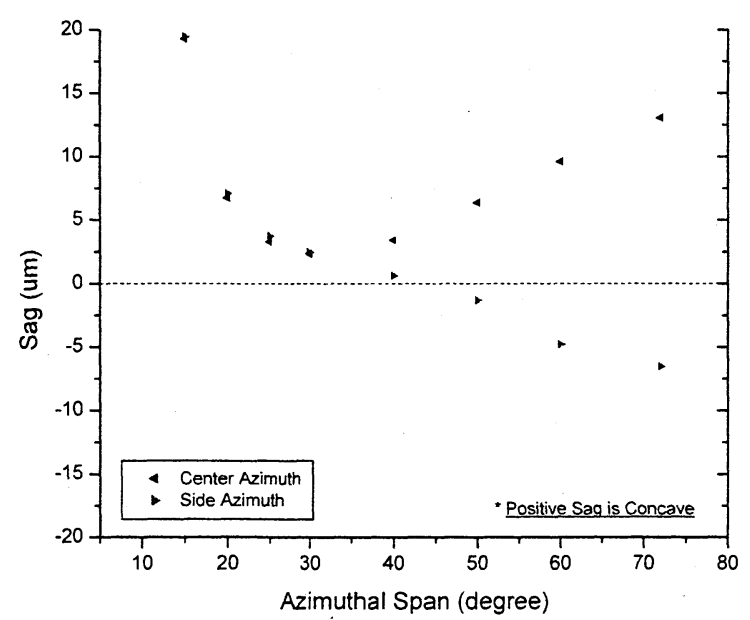

Fig. 4. Optimization of azimuthal size of mirror with a fixed diameter $(489 \mathrm{~mm})$. 3-point mount. Support at one end with $22.5 \%$ of azimuthal length from edges; that is, middle portion is $55 \%$. Support at the other end is at the center of the end. Sag at edge becomes more convex as mirror is larger. Middle profile goes through a change in sign of sag, due to anticlastic bending for the conical mirror. Minimal sag is around 30 degree.

For the question of scaling with radius, we repeat the calculation for a series of radii: $R=150,200,245,350,475$ and 650 $\mathrm{mm}$. This covers the full range of mirror radii in the Constellation-X baseline design. Generally, sag is small in the center of the mirror and increase towards the edge, except for the "plate-like" cases. The optimal angle is expected to 
scale slower than the zero-th power of the radius---a mirror with a fixed angular span is more easily distorted for a larger radius. In fact, the optimal angular span is found to scale with $1 / R$. That is, the optimal physical size of the mirror is roughly constant. In numerical terms, optimal angular size for $R=245$ is $\sim 30^{\circ}$, for inner mirror with $R=150$ it is $\sim 50^{\circ}$, for outer mirror with $R=650$ it is $\sim 10^{\circ}$. Overall scaling can be summarized as follows: optimal angular size $S_{0} \sim 360^{\circ}(20$ $\mathrm{mm} / R$ ). The tilts of the axial profiles generally increase from the center to the edges. Tilt can be large at edge for large mirror, either for mirror with a large radius or with a large angular span. More study is needed to understand DeltaDelta- $R$ effect if the mirror is to be forced to be fixed, at the boundary, to its proper radius.

\section{SPRING "MATTRESS" MOUNT}

To support the mirror at more than 4 contact points, a more compliant contact than rigid hard contact is needed in order not to over-constrain the mirror. This is based on the reality that we are not able to position all the contact point to match the geometry of the mirror to sub-micron precision. In addition, the geometry of the undistorted mirror is not known $a$ priori. We attempt to achieve this with a bed of very soft springs, in the form of coils. To illustrate the concept, we consider a simple beam on a linear array of springs. The small deflection of the beam due to its own weight under gravity is described by

$$
\frac{d^{2}}{d x^{2}}\left(E I \frac{d^{2} w}{d x^{2}}\right)=-\mu g
$$

where $x$ is the beam "axial" direction, $w$ is the deflection in the vertical, $E$ the Young modulus, $I$ the cross-sectional moment of inertia, $\mu g$ the gravity loading of the beam per unit length, $\mu$ is the mass per unit length. This equation can be integrated with boundary loading at the spring locations to obtain the deflection. The spring is idealized as linear spring $F=k w$, with spring constant $k$ set by a fixed overall compression of springs in static equilibrium, $k \propto 1 / N$, where $N$ is the number of springs. It is easily found that the resulting deflection depends on the distribution of springs. We consider three distributions of springs, all of them uniform distributions but with different overhang, as depicted below. The result, which is verified with finite-element models, is shown in figure 5.

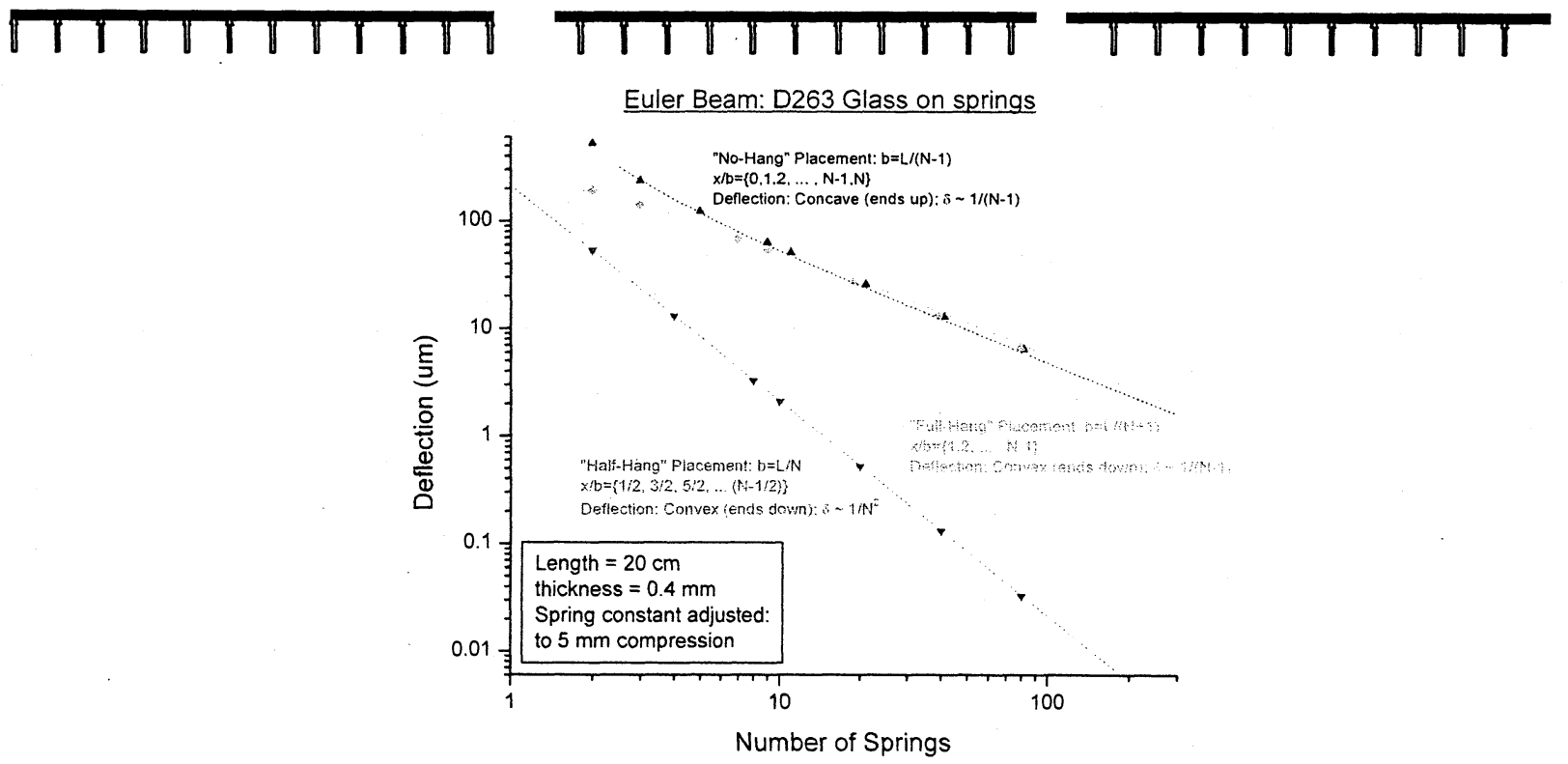

Fig. 5. Dependence of deflection of mirror on the number of springs. Linear models of beam deflection on a linear array of uniformly distributed springs are calculated. Deflection critically depends on the amount of overhang for larger number of springs than a few. Top illustration shows the three overhang conditions: 1. No overhang. 2. Half hang. 3. Full hang. Bottom graph shows the result of maximum deflection for a beam or plate and its scaling with the number of springs. Expect for case 2, half-hang, which is the special condition where the mirrors are critically "balanced", the dependence goes like $1 / N^{2}$. The deflection shown here is offset by the initial fixed compression of springs. 
The results shown in figure 5 are from finite-element modeling as well as from direct integration of the equation above. The finite-element models are for a thin plate of $200 \mathrm{~mm}$ long and $0.4 \mathrm{~mm}$ thick. The analytical result is from symbolic integration of the beam-bending equations, with Mathematica, up to $N \sim 20$. The springs are identical linear springs with the spring constant adjusted to provide a fixed static vertical compression of $5 \mathrm{~mm}$. We note that the compression is relatively large compared to the original uncompressed size of the coil and perfect linearity is questionable. We have measured the linearity of the springs and found them to be quite linear. More important, for the mattress to perform statically, the spring does not have to have strict linearity for the whole range of compression. An effective spring constant $k$ may be represented by the local gradient $k=d F / d z$. A relatively large compression of a few mm by design provides the tolerance that a minor error, say, $0.1 \mathrm{~mm}$, in the machining of supporting bed or the original extent of the spring will not be significant.

The result shows that for a flat plate or a beam, with proper overhang, the sub-micron deflection can be achieved with tens of springs in a row. We proceed to model a full mattress/cradle system for a conical segment of mirror. Again, similar to the flat case, we prescribed a fixed overall vertical compression. An actual proto-type model was made and used for metrology ${ }^{5}$, as shown in figure 6 below. The system illustrated below is for 12 rows of coils, each having 18 mylar loops with $1 \mathrm{~cm}$ diameter. These coils are sufficiently soft and the spring constant is $\sim 0.4 \mathrm{~N} / \mathrm{m}$, giving an overall vertical compression of $\sim 5 \mathrm{~mm}$.

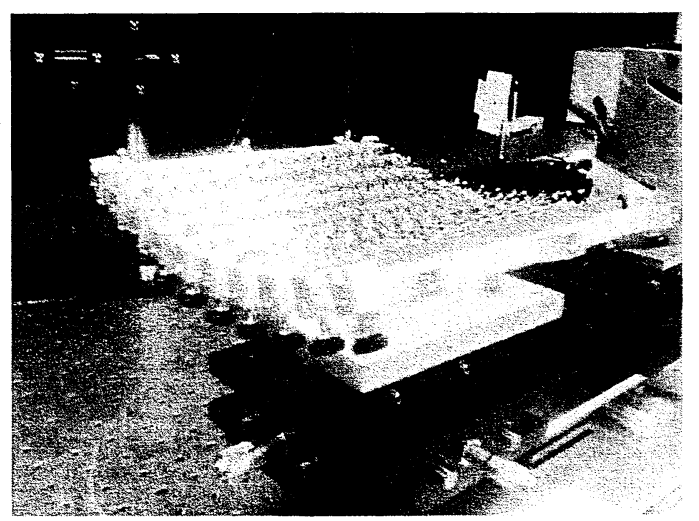

Fig. 6. A model of the mattress/cradle system for mirror support. The mattress, with removable rows of coils', sits on a cradle made of the same glass material. This ensures minimal mismatch in coefficient of thermal expansion. Individual coils can also be adjusted in tilt angle by the screws at the end of the system, for alignment purpose. The whole system is seated on tip/tilt and linear stages.
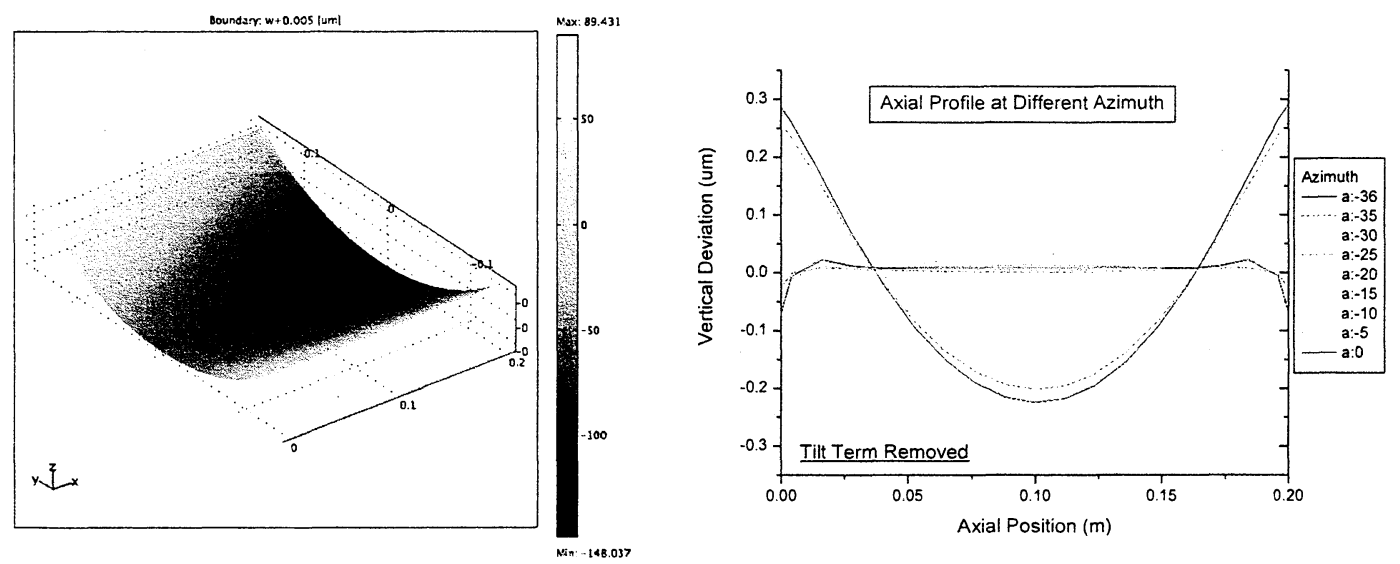

Fig. 7. Displacement of mirror on mattress, supported by a cradle. Angular span of the mirror is 72 degree. (Left) Overall vertical depression of the mirror, offset by the $5 \mathrm{~mm}$ overall compression by design. Most of the displacement is due to a change in tilt angle of the mirror---the mirror is of conical shape, with one axial end larger, and therefore heavier than the other end---resulting in axial asymmetry. (Right) The axial profiles at various azimuths, with tilt-term removed. The axial profile is distorted only by a small fraction of microns, demonstrating the overall effectiveness of the mirror in terms of axial sag. 
Figure 7 shows the displacement of a $244.5 \mathrm{~mm}$ radius, 72-degree conical mirror with a small ( $\sim 1 \mathrm{micron})$ sag, on a mattress, supported by a perfectly rigid cradle, after a constant built-in offset of $5 \mathrm{~mm}$ is removed. The springs are linear springs and we recall that only a local effective $k$ is significant here for static consideration. The reaction of spring on the mattress is assumed to be normal to the mirror surface, but it is demonstrated that additional tangential component does not affect the result significantly. The range of displacement is large $(\sim 200 \mu \mathrm{m})$ but the displacement is mostly a constant displacement and a tilt. The tilt comes from the axial asymmetry of the mirror---it is a conical instead of cylindrical. Due to non-vertical nature of the spring's action, the mirror can no longer retain its circularity with the same radius. It is demonstrated, not shown here, that the number of rows of coils, and their placement with respect to the mirror (and their distributions) are important variables for this circularity. The azimuthal deviation is consistent with simple beam models. Further study to understand and optimize "delta-delta-R" in terms of placement of rows of coils is underway.

In the axial direction, the axial sag is significantly smaller than the boundary-point mounts described in Section 2.1 to 2.3 , thus demonstrate the potential of the mattress/cradle system. We note that in our implementation of springs, the action of coils on the mirror is not actually point-like. It is rather large instead with the significant $5 \mathrm{~mm}$ compression. Even though additional tangential force from the flattened coils may not be significant, the non-zero dimension of the contact may change the effective positions of the spring action. These remain to be investigated. Of equal importance in the performance of the mattress system are: uncertainty in radius of cradle, uncertainty in coil positions and uncompressed levels. As shown for a linear beam on springs, unbalanced placement of mirror on the mattress may cause large and different deviation in the axial direction, especially at the edge. In fact, depending on the placement or overhang, the concave-ness or convex-ness of the axial profile at the mirror edge can be different.

\section{MIRROR TRANSFER AND BONDING}

Beyond mounting a mirror temporarily for metrology, the mirror needs to be transferred to a more rigid structure and be bonded to it. We investigated this process with a proto-type Cradle Alignment Transfer System (CATS), shown in figure 8. The system consists of a rigid structure, in a form of a cube, where two T-rails are mounted on the sides near the top. A mirror, supported by the mattress/cradle can be raised and placed into the slotted T-rails, where the mirror will be bonded by epoxy encapsulation. The details of this process will be discussed elsewhere.

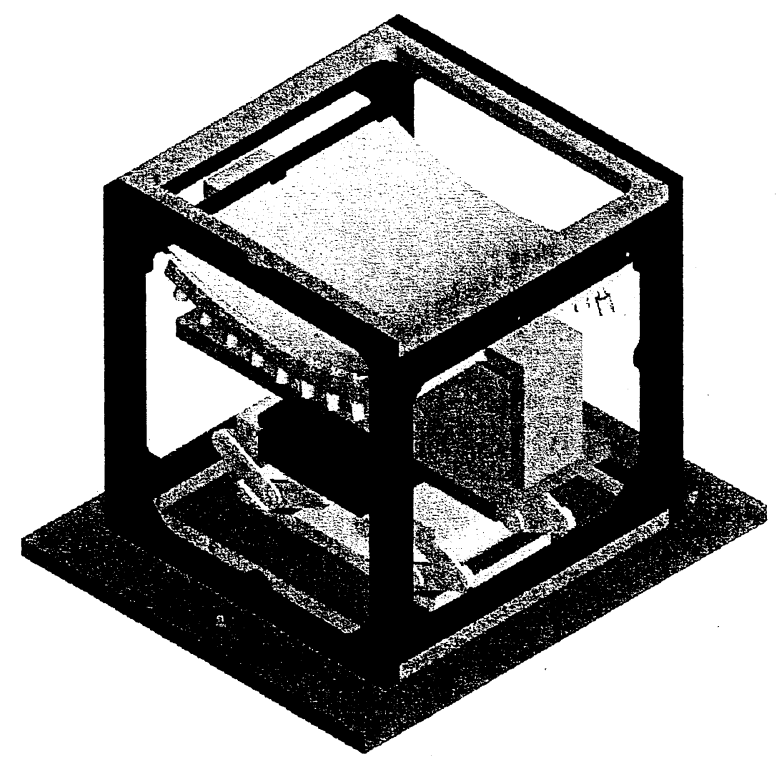

Fig. 8. The Cradle Alignment Transfer System (CATS) for transfer and bonding of a mirror to a rigid structure. Both the framed structure and the cradle are made of materials of compatible coefficient of thermal expansion for thermal stability. 
With bonding to a rigid structure, thermal stress on the mirror from incompatible structural material cannot be neglected. In the design of the CATS system, CTE (coefficient of thermal expansion) compatible material such as Ti metal is used to minimize the thermal strain on the glass mirror. The CTE-matching is not perfect and there remains a difference of about $1-2 \times 10^{-6} /{ }^{\circ} \mathrm{C}$, but glass is not a viable structural material in this case. The present experimental environment is expected to have a $1-2{ }^{\circ} \mathrm{C}$ excursion. We note that for an $L=200 \mathrm{~mm}$ long mirror, the linear strain is $\alpha T L \sim 0.5 \mu \mathrm{m}$, where $\alpha$ is the difference of CTE between the glass mirror and its bonding structure, $T$ is the temperature variation. In a situation when the mirror is compressed, if length is preserved under such a buckling condition, a flat piece of glass will have a buckled sag of $\sim(3 \alpha T / 8)^{1 / 2} L \sim 0.2 \mathrm{~mm}$, which is large. This gives an upper limit since the cylindrical geometry of the mirror and its elasticity will reduce the length-preserving sag. At any rate, these estimates indicate that thermal stress may be significant and its effect demands a more detailed modeling.
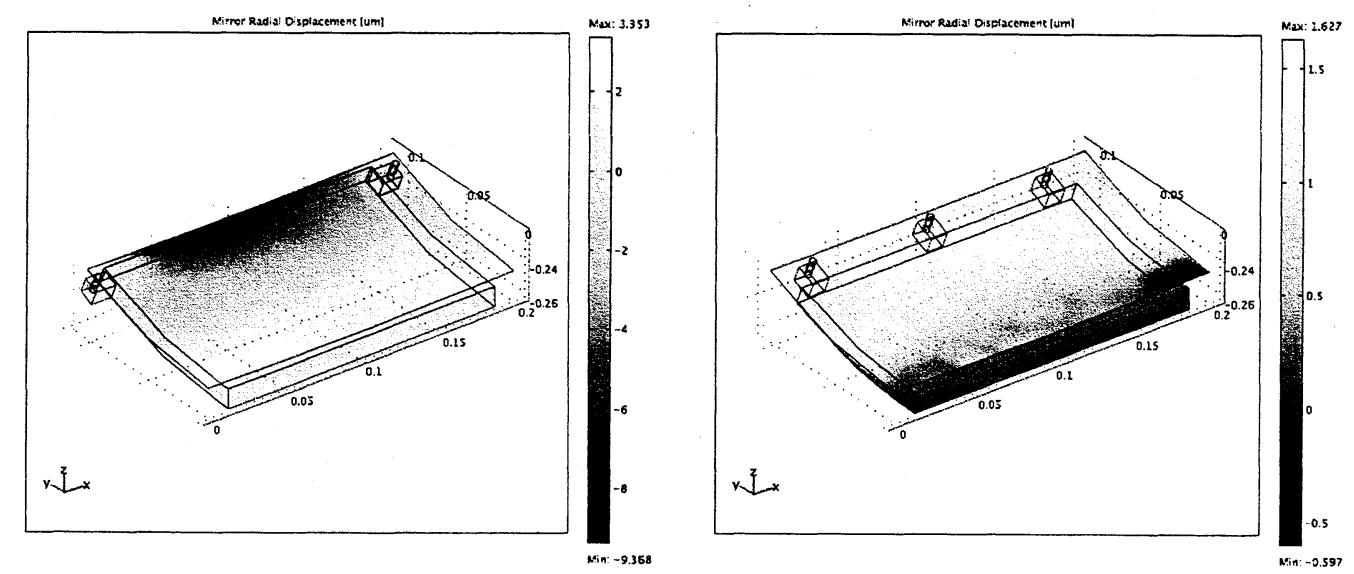

Fig. 9. Simulation of a mirror held by 4 or 6 points with a thermal change of $-2^{\circ} \mathrm{C}$. Gravity is as well as mattress spring reaction is removed from the system in order to study purely the thermal effect. The cradle is made with CTE-matching glass, but the mirror is held by tabs and adjustment screws that are made of stainless steel. Contact at the adjustment screws are areal instead of an idealized point, implying constrain also in the bending moment of the mirror at those boundaries. (Left) The mirror is held at 4 points near the corner of the mirror. (Right) The mirror is held at 6 points along the 2 edges of the mirror. Only half of the mirror is modeled, by symmetry.

Thermally induced distortion, of a conical mirror with the same dimension as described previously, is modeled for 2 different boundary conditions: 1 . Bonding at 4 positions near the corners of the mirror; 2 . Bonding at 6 positions at the edges of the mirrors. The 3 bonds at each edge are assumed to have no error in levels and do not over-constrain the mirror in zero $\Delta T$. The mirror is bonded to adjustment screws with a $2 \mathrm{~mm}$ radius, which are threaded through tabs rigidly mounted on a cradle. The boundary condition on the mirror is an areal one and thus has also impacted bending moment to the mirror. The cradle itself is made of the same glass and is therefore CTE-matching. It has small gravity sag of about $0.1 \mu \mathrm{m}$ in the present support system. In order to machine the posts and tabs (about 0.5 inch in length), they cannot be made of glass. Steel was chosen for experimentation and therefore can stress the mirror thermally in unstable environment. It turns out that a temperature of $\sim-2^{\circ} \mathrm{C}$ was observed.

Finite-element model shows that thermal effect in corner-bonded mirror is important at $\Delta T \sim-2^{\circ} \mathrm{C}$. About $4 \mu \mathrm{m} /{ }^{\circ} \mathrm{C}$ of axial sag can be induced at mirror edges (figure 9). To study purely the thermal effect, the action of mattress and gravity is removed (gravity is kept for the cradle just to hold it down for mechanical stability.) There is smaller sag in the middle of the mirror and mildly convex profiles for the azimuths in between the middle and edges. The model is roughly consistent with observation. Thermal effect in axial profile is much reduced with the edge-bonded mirror by about an order of magnitude in axial sag at the edge of the mirror. Bending is then largely azimuthal, as expected. The edgebonding tabs and screws are an angle of $25^{\circ}$ and $65^{\circ}$ to the horizontal and are of comparable length. This provides a partial cancellation of thermal strain in the horizontal direction. More detailed study and design (dependent of mirror angular size) is underway to provide better athermalization of the system.

Additional models are done for mirror with the CATS' cube (see also figure 10) using NASTRAN finite-element software. Mirror distortions under a $+1{ }^{\circ} \mathrm{C}$ bulk temperature change are calculated for the mirror mounted in the cube. The cube is made of $\mathrm{Ti}$ or Macor and has a $\sim 1-2 \times 10^{-6} /{ }^{\circ} \mathrm{C}$ in CTE mismatch. Two boundary conditions are considered 
for a mirror with $242.5 \mathrm{~mm}$ radius. Mirror was held at the 6 mounting points. Bond points of the mirror were connected to the cube in 2 different ways to envelope over constrained and least constrained conditions: 1 . BC5, in which all 6 degrees of freedom are constrained. 2. BC3, in which the center point is constrained but outside 4 points are only constrained in radial downward direction. The result is passed on to an optical code to evaluate its effect on the imaging. The result is summarized in the table below.

\begin{tabular}{|l|l|c|c|c|c|}
\hline \multicolumn{1}{|c|}{ Case } & \multicolumn{1}{|c|}{ Boundary Condition } & \multicolumn{2}{c|}{ Ti Cube } & \multicolumn{2}{c|}{ Macor Cube } \\
\hline & & $\begin{array}{c}\text { Surface } \\
\mathrm{rms}(\mu \mathrm{m})\end{array}$ & $\begin{array}{c}\text { Image } \\
\mathrm{HPD}(\text { (“) }\end{array}$ & $\begin{array}{c}\text { Surface } \\
\mathrm{rms}(\mu \mathrm{m})\end{array}$ & $\begin{array}{c}\text { Image } \\
\mathrm{HPD} \text { (“) }\end{array}$ \\
\hline BC-5 & 6 points constrained in 6 degrees of freedom & 0.63 & 6.8 & 0.41 & 4.7 \\
\hline BC-3 & $\begin{array}{c}\text { Center points: constrained in 3 translational } \\
\text { degrees of freedom; Corner points: } \\
\text { constrained along radius; free to move } \\
\text { along axis and tangent }\end{array}$ & 0.43 & 0.15 & 0.88 & 0.35 \\
\hline
\end{tabular}

\section{DYNAMICAL CONSIDERATION}

To assess the dynamical effect a mirror mounted in the CATS' cube, we performed an eigenfrequency analysis of the mirror in cube. We simulated the mirror edge-bonded at 6 points to the cube. Different boundary conditions are considered. Three cases are listed in the table below. Boundary conditions BC- 3 and BC-5 are the same as described before. Condition BC-1 is the less constrained boundary condition, where the 2 center points are held in 3 translational degrees of freedom, but the 4 corners are held only in the radial direction. The table listed the frequencies of the fundamental normal mode.
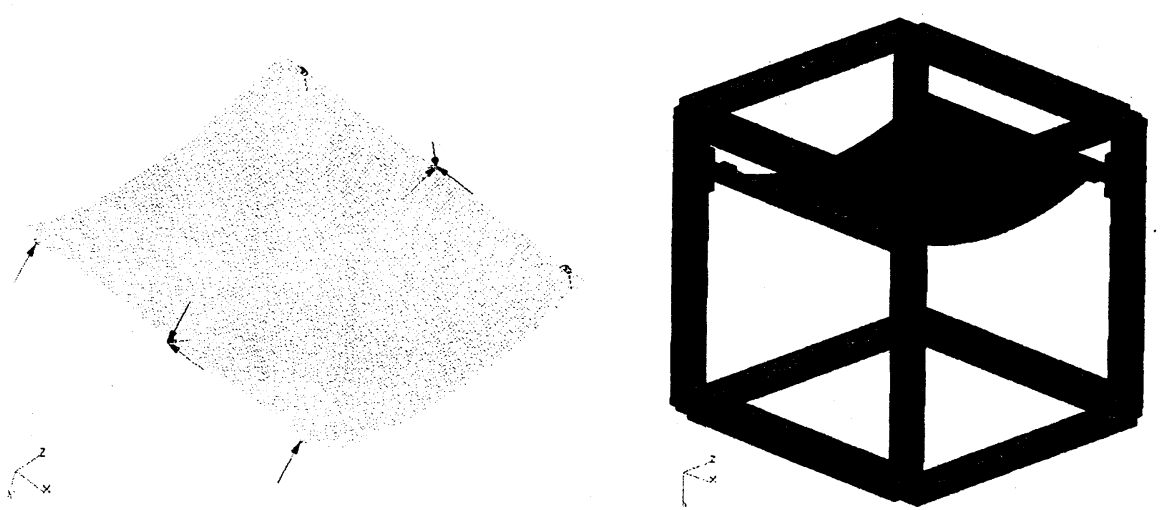

Fig. 10. Simulation condition for dynamical consideration. (Left) The mirror with its 6 boundary locations. Boundary conditions are indicated with arrows for case BC-3. (Right) Illustration of mirror in CATS' cube frame.

\begin{tabular}{|l|l|c|}
\hline Case & Edge Boundary Conditions & $\begin{array}{c}\text { Fundamental Frequency } \\
(\mathrm{Hz})\end{array}$ \\
\hline $\mathrm{BC}-3$ & $\begin{array}{l}\text { Center point held in 3 degree of freedom, corners held } \\
\text { only in Radial direction }\end{array}$ & 31.8 \\
\hline $\mathrm{BC}-1$ & All points held in 3 translation degree of freedom & 79.4 \\
\hline $\mathrm{BC}-5$ & All points held in all 6 degree of freedom & 117.3 \\
\hline
\end{tabular}




\section{SUMMARY}

In summary, we have addressed gravity distortion from mounting the thin Constellation-X mirror segments. Holding a mirror with its axis horizontal and mirror facing upward (or downward) at a small number of points, such as 4, at the boundary is not desirable. We found that the mattress may provide a solution to a temporary mount in this orientation. The axial figure deformation is generally small but there is variation of tilt across the azimuths. Better design of spring placement and distribution should be able to minimize the variation. Mounting the mirror in the vertical orientation provides another option for mirror test outside the x-ray beam line. In this orientation, the gravity is no longer perpendicular to the mirror surface and the distortion is reduced. Work is in progress especially for the newly design "tree"-mount and "suspension"-mount. Thermal stress from CTE mismatch of the mirror and housing materials is important and the requirement is stringent when the mirror is rigidly bonded to its housing structure. Further study is underway in this aspect.

\section{REFERENCES}

1. N. E. White, et al. "Constellation-X Mission Implementation Approach and Status", Proc. SPIE, Vol. 6266, 626662 (2006).

2. R. Petre, et al. "Constellation-X Spectroscopy X-ray Telescopes: Recent Technology Development", Proc. SPIE, Vol. 6266, 62661Q (2006)

3. W. Zhang, et al. "Development of Light-weight Mirrors for the Constellation-X Mission", Proc. SPIE, Vol. 6266, $62661 \mathrm{~V}(2006)$

4. W. Zhang, et al. "Constellation-X mirror technology development status and plan", Proc. SPIE, This volume (2007)

5. T. Hadjimichael, et al. "Alignment and integration techniques for mirror segment pairs on the Constellation-X telescope", Proc SPIE, This volume (2007) 